\title{
Miniature magnetic devices for laser-based, table-top free-electron lasers
}

\author{
T. Eichner, ${ }^{1}$ F. Grüner, ${ }^{1}$ S. Becker, ${ }^{1}$ M. Fuchs,${ }^{1}$ D. Habs,${ }^{1}$ R. Weingartner, ${ }^{1}$ U. Schramm,,${ }^{1,2 *}$ H. Backe, ${ }^{3}$ \\ P. Kunz, ${ }^{3}$ and W. Lauth ${ }^{3}$ \\ ${ }^{1}$ Department für Physik, LMU München, 85748 Garching, Germany \\ ${ }^{2}$ Forschungszentrum Dresden-Rossendorf FZD, 01314 Dresden, Germany \\ ${ }^{3}$ Institut für Kernphysik, Universität Mainz, 55099 Mainz, Germany
}

(Received 30 March 2007; published 17 August 2007)

\begin{abstract}
Truly table-top sized radiation sources based on compact laser-plasma accelerators require compact and strong focusing devices and efficient short-period undulators. Complementing our recent theoretical work on the feasibility of a table-top FEL, we here present the design and successful experimental characterizations of a $5 \mathrm{~mm}$ period length undulator and miniature quadrupole magnets with field gradients of the order of $500 \mathrm{~T} / \mathrm{m}$.
\end{abstract}

DOI: 10.1103/PhysRevSTAB.10.082401

\section{INTRODUCTION}

Over the past few years, laser-plasma acceleration of electrons [1-3] has rapidly advanced into a controllable technique $[4,5]$ that is now widely discussed for first applications. Appealing features of laser-plasma accelerators are the mm-scale size that is required for the generation of $\mathrm{GeV}$ electron energies [4] and the high peak currents of up to $100 \mathrm{kA}$ combined with short bunch lengths on the $10 \mathrm{fs}$ scale [6]. Especially the latter suggests the application of laser-accelerated electron bunches as drivers for secondary light sources, either via incoherent synchrotron radiation [7], undulator radiation, or coherent FEL (free-electron laser) radiation [8].

Although the laser-plasma accelerator can be regarded as being intrinsically table-top, the goal of a true table-top concept requires the development of both compact shortperiod undulators and miniature focusing devices. In a recent design study [8], our group pointed out the feasibility of the operation of a laser-plasma accelerator driven table-top FEL. Thus, the principle aim of this paper is to give experimental evidence that the crucial components that have to be used to control the propagation of the electron beam can be sufficiently miniaturized. We discuss the case of quadrupole lenses and undulators that represent the most fundamental optical elements, bridging the gap between conventional well-established accelerator technology and novel laser-plasma accelerators. A compact sample scenario is illustrated in Fig. 1. An energetic electron bunch is emitted from a near pointlike source with a divergence of a few mrad. A doublet of miniaturized highgradient quadrupole magnets is used for the imaging of the source to the compact undulator only about one meter downstream. The image of the beam waist is considerably enlarged as a low divergence is required for the generation of narrow-band undulator radiation.

\footnotetext{
*Corresponding author. u.schramm@fzd.de
}

PACS numbers: 41.85.Lc, 41.60.Cr, 41.75.Jv, 52.38.Kd

FEL operation is usually based on a planar undulator [9]. Such an arrangement of alternating magnetic dipole fields forces the electron bunch on a sinusoidal trajectory. The bunch can thus couple with a copropagating radiation field. Because of the dispersion of the dipole fields, the induced energy modulation yields a current modulation, called microbunching. It expresses the fact that the electrons are grouped into small bunches separated by a fixed distance that resonantly coincides with the period length of the radiation field. Consequently, the radiation field can be amplified coherently. Lacking an initial resonant radiation field, a seed may also build up from spontaneous incoherent emission [10] in the SASE (self-amplification of spontaneous emission) process.

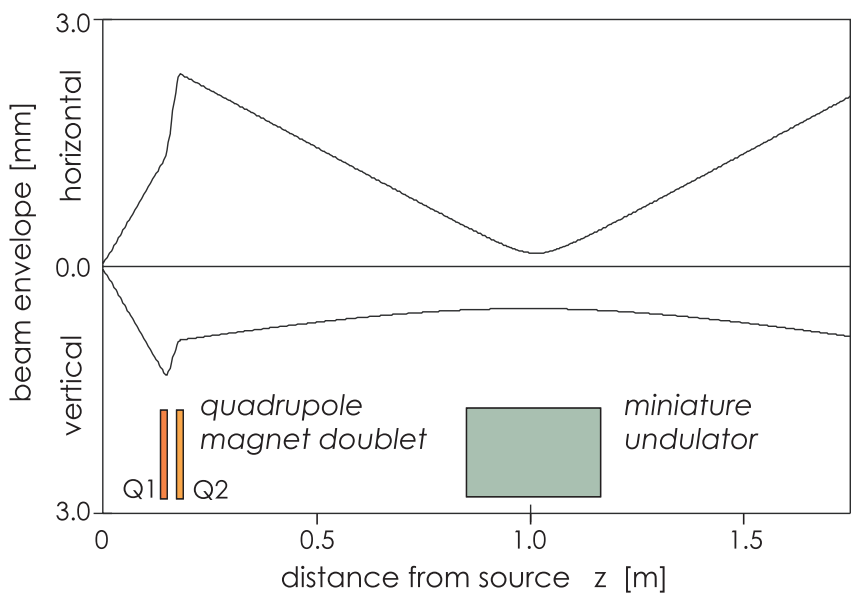

FIG. 1. (Color) Development of the horizontal (upper curve) and vertical (lower curve) beam envelopes of a $150 \mathrm{MeV}$ laseraccelerated electron beam. The beam originates from a source of radius $1.3 \mu \mathrm{m}(z=0)$ at an exaggerated maximum divergence angle of $8 \mathrm{mrad}$. It enters a focusing quadrupole doublet (field gradient $500 \mathrm{~T} / \mathrm{m}$, individual lengths of the magnets $L_{Q 1}=17 \mathrm{~mm}$ and $L_{Q 2}=15 \mathrm{~mm}$ ) and is focused into the miniature undulator $\left(L_{u}=300 \mathrm{~mm}\right)$. 
Restrictive conditions have to be fulfilled for the amplification of radiation that, simply speaking, express the need for an overlap between the electron beam and the radiation field in all respects. The radiation bandwidth, and correspondingly the electron energy spread, has to be smaller $\left(\Delta \lambda_{1} / \lambda_{1}=2 \Delta \gamma / \gamma<2 \rho\right)$ than the so-called Pierce parameter $\rho$ [10]. In large-scale FELs this parameter is typically of the order of $10^{-4}-10^{-3}$ while for laser-based FELs it can be 1 order of magnitude larger, especially due to the high peak currents [8]. Tolerances of the magnetic field distribution of the undulator are accordingly determined by the Pierce parameter, as will be discussed quantitatively later. A well-defined transport of the electron beam through the undulator has to ensure a divergence matched to the emission characteristics.

Besides the most demanding scenario of the table-top FEL [8] for which the components presented here have been developed, miniaturized lenses and undulators will have numerous applications in conventional as well as laser-driven accelerators. High gradient and thus short focal-length magnetic lenses have the potential for generating focal spots in the sub-micron range. These could be used as pointlike sources of hard radiation for phase contrast imaging with unprecedented transverse coherence [11], for compact Thomson scattering sources [12], or for the localized deposition of high radiation doses. Short focal-length lenses are further mandatory for the compact relay imaging of electron bunches between consecutive acceleration stages in cascaded laser-plasma acceleration schemes. Especially the postacceleration of dense bunches of electrons, first accelerated in the bubble regime [6,13], requires careful matching of the bunch density to the lower plasma density in a plasma wakefield accelerator. Such scenarios might help to increase the energy and improve the energy spread of bubble accelerated dense bunches and are thus of vital interest for table-top FEL projects.

In the following sections, detailed descriptions of the design of the miniaturized magnetic devices and their commissioning at the Mainz electron accelerator MAMI are given. In the last section, applications of undulators in radiation generation and diagnostics of laser-plasma accelerated beams are discussed.

\section{HIGH FIELD MINIATURE UNDULATORS}

The generation of hard undulator radiation of wavelength

$$
\lambda_{n}=\frac{\lambda_{u}}{2 \gamma^{2} n}\left(1+\frac{K^{2}}{2}+\gamma^{2} \theta^{2}\right)
$$

with comparatively low electron energies $\gamma$ relies on the reduction of the undulator period length $\lambda_{u}$ from the usual $\mathrm{cm}$ scale to a mm scale, $\theta$ standing for the observation angle and $n$ for the harmonic number of the emitted radiation. The performance of such an undulator largely depends on its deflection strength that is described by the normalized undulator parameter

$$
K=\gamma \theta_{e}=\frac{e \lambda_{u} B_{y, 0}}{2 \pi m_{e} c} \approx 0.93 \lambda_{u}[\mathrm{~cm}] B_{y, 0}[\mathrm{~T}]
$$

$\theta_{e}$ depicting the maximum electron deflection angle. For most undulator types, the amplitude of the magnetic field on axis $B_{y, 0}$ can be maintained during miniaturization when the ratio of the gap height $g$ to the period length $\lambda_{u}$, ideally being of the order of $g / \lambda_{u} \sim 0.3$, is preserved [9]. Thus, a reduction in $\lambda_{u}$ implies an at least similar reduction in $g$ and both will finally set limits to the miniaturization.

\section{A. Design and manufacturing}

For the construction of short period length undulators, different techniques have to be considered [9] starting with the well-established Halbach design [14]. In this pure permanent magnet (PPM) design, the field of the alternating magnet poles is closed by additional magnets rotated by $90^{\circ}$, similar to the case of the PPM quadrupoles, discussed in the next section. In contrast, the key of a hybrid design is to not only guide the field of permanent magnets from one period of the undulator to the next, but to concentrate and further increase the field in ferromagnetic plates that form the alternating poles of the undulator. This design is illustrated in Fig. 2 emphasizing the field concentration in the $x-y$ pole plane. Typically, an increase of the peak field on axis $B_{y, 0}$ by $20 \%$ can be achieved as compared to the PPM type. However, due to the nonlinearity of the approach, this value strongly depends on design details. Modern ferromagnetic materials such as tempered Co-Fe alloys (e.g., vacoflux 50 [15]) provide high saturation field strengths of the order of $2.3 \mathrm{~T}$ at room temperature, where the maximum remanent field of $\mathrm{Nd}_{2} \mathrm{Fe}_{14} \mathrm{~B}$ magnets reaches values of 1.2-1.4 T. The hybrid design allows for shorter periods as the ferromagnetic plates can be chosen thinner and ultimately, on the $\mathrm{mm}$ scale, manufactured thinner than sintered permanent magnet plates. In decreasing the period to the few-mm scale, however, further aspects have to be regarded. Magnet material with high coercivity has to be carefully chosen in order to withstand the magnetic shear forces, as irreversible demagnetization starts at moderate temperatures of $\sim 60-80{ }^{\circ} \mathrm{C}$.

As a compromise between the design goal of a miniature in-vacuum hybrid undulator of a short-period length of few $\mathrm{mm}$, a gap of at least $g=1.5 \mathrm{~mm}$, and a field of the order of $B_{y, 0}=1 \mathrm{~T}$, and, on the other hand, tolerable magnet stability and handling, a period length of $\lambda_{u}=5 \mathrm{~mm}$ has been chosen for the prototype presented here. The design (Fig. 2) was inspired by a similar design of two short $12 \mathrm{~mm}$ period precision undulators built in Mainz [16]. Its total length amounts to $N_{u}=59.5$ periods or $\sim 30 \mathrm{~cm}$ as depicted in the photograph in Fig. 3. Two half periods with approximately half the field strength at the ends [17] 


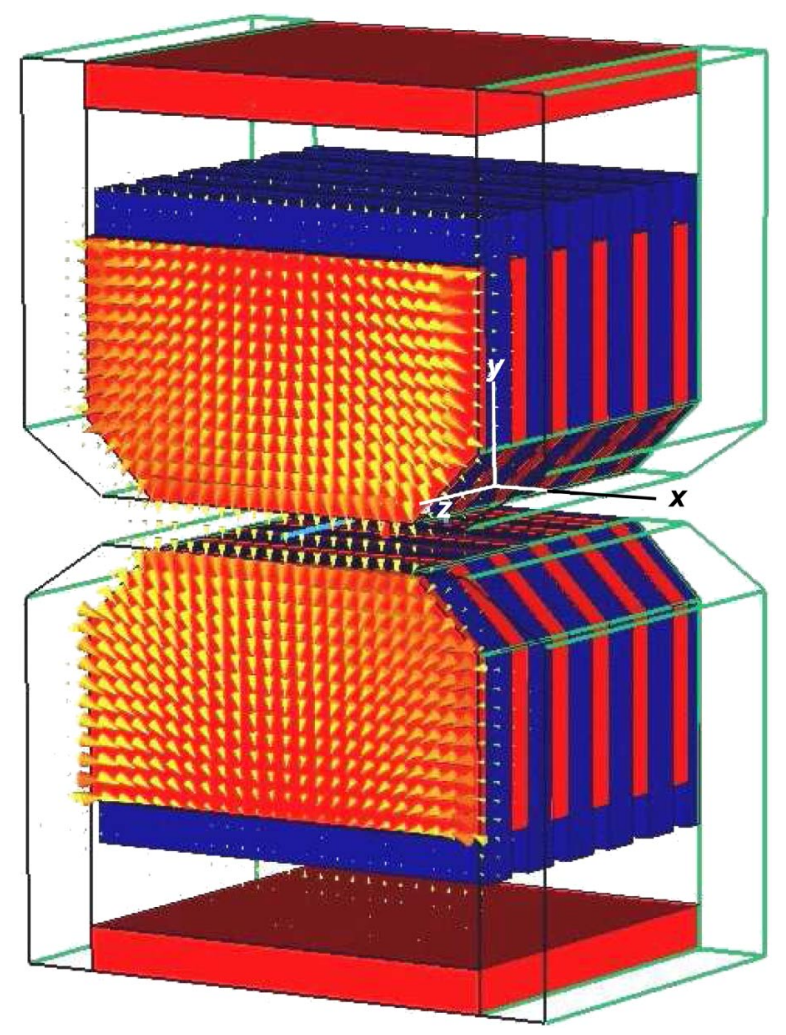

FIG. 2. (Color) Schematic view of the hybrid design of the miniature undulator, consisting of alternating plates of permanent magnets (blue), polarized parallel and antiparallel to the undulator axis $z$, and ferromagnetic material (red). In the front $x-y$ plane arrows indicate the guiding of the magnetic field inside the ferromagnetic plates toward the undulator gap (simulation CST EM Studio). The transparent frames show the rail structure supporting the magnet blocks, also visible in the photograph in Fig. 3.

are added for a near deflection-free and straight transmission of an electron beam as discussed below.

Precision glued stacks of $1 \mathrm{~mm}$ thick Vacoflux 50 plates and $1.5 \mathrm{~mm}$ thick Vacodym $764 \mathrm{HR}$ [15] magnets of 4 periods length form mounting units of the size of the order

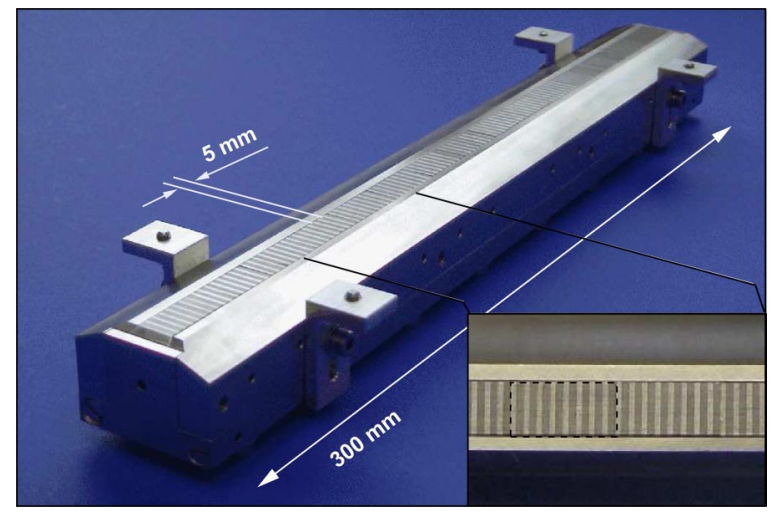

FIG. 3. (Color) Photograph of the lower half of the 60 period planar undulator after the first preliminary assembly. In the inset, the precision glued magnet stacks are visible (dashed box). of a $\mathrm{cm}^{3}$ as depicted in the inset of Fig. 3. Overall mechanical rigidity is guaranteed by nonmagnetic profiles anchored in the outer ferromagnetic yokes on either side of the magnet stacks (see Figs. 2 and 3). These profiles form a rail that holds the magnet stacks in place with a precision of the order of $10 \mu \mathrm{m}$. However, this precision on the percent level of the gap width implies deviations from the ideal field on axis $B_{y, 0}$ on the same level. Thus, after the final assembly of the full system, the gap surface of the undulator was carefully finished by grinding.

At the yoke side of the magnet stacks, the length of the magnets exceeds that of the ferromagnetic plates (see Fig. 4) in order to avoid a short-circuiting of the field between neighboring plates and thus provide the high tip field at the gap side of the poles of about $2 \mathrm{~T}$. At the sides of the stacks in the $y-z$ plane, this option could not be chosen to further increase the field as driving the plates further into saturation reduces the ability for an individual fine adjustment. This fine adjustment of the gap field can in principle be performed by a defined coupling of the individual plates to the yoke via ferromagnetic screws, similar to the technique applied for the tuning of the end plates, that is visible in Fig. 4.

The inset of Fig. 5 shows the distribution of the magnetic field perpendicular to the undulator plane $B_{y}(z)$ along the undulator axis $z$. It was measured after the grinding of the magnet surfaces by moving the undulator along a station-

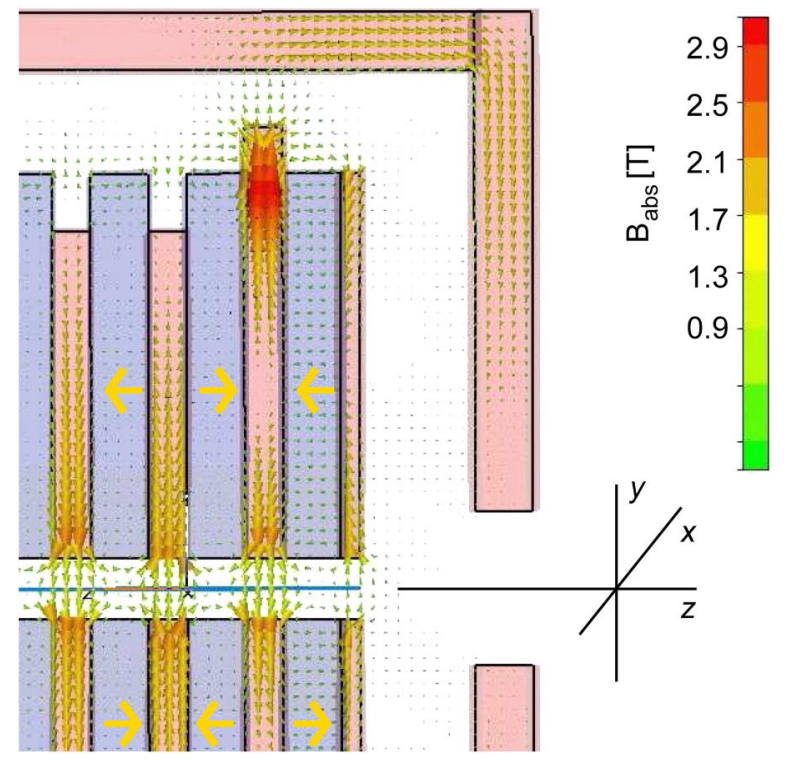

FIG. 4. (Color) False color arrows indicate the direction and strength of the magnetic $B$-field in the $y-z$ plane of the hybrid undulator. The field at the pole tips amounts to $\sim 2 \mathrm{~T}$, the corresponding $B_{y, 0}$ field on axis to $\sim 0.9 \mathrm{~T}$. In the simulation, the magnetization of the thin permanent magnets, indicated by yellow arrows, was set to $1.3 \mathrm{~T}$. Slight tuning of the field on axis can be achieved by coupling the upper part of the ferromagnetic plates to the outer yoke structure, as displayed for the tuning of the end plates. 


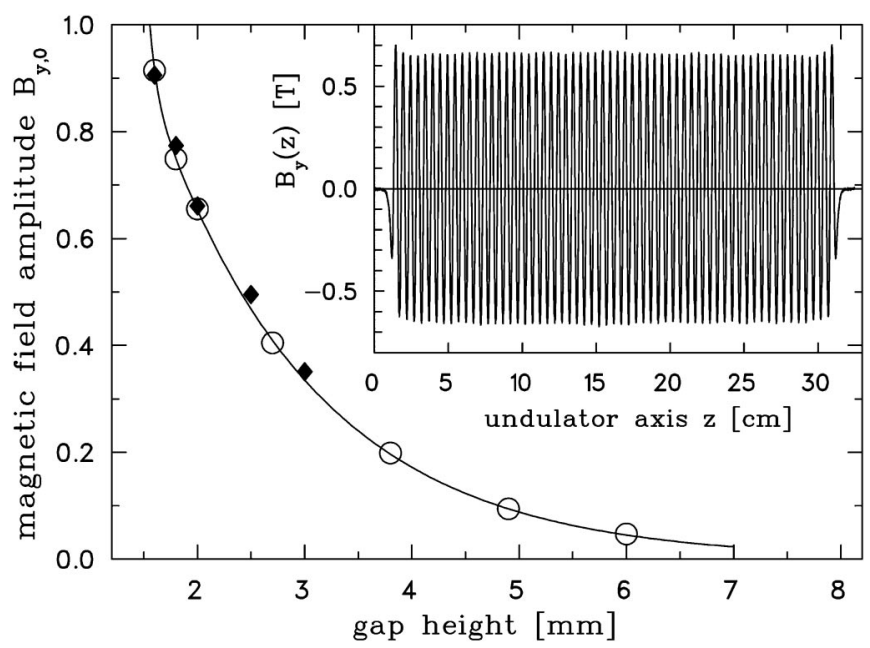

FIG. 5. Hall probe measurement of the magnetic field along the axis of the miniature undulator $B_{y}(z)$ for a gap height of $2 \mathrm{~mm}$ (inset). Measured peak values of the field $B_{y, 0}$, corrected for the finite probe size of $0.75 \mathrm{~mm}$ diameter by $6 \%$ (rhombs), are compared to simulation results (CST EM Studio, open circles) as a function of the gap height $g$. The line shows a double exponential fit according to Ref. [9].

ary precision Hall probe with $0.75 \mathrm{~mm}$ head diameter. Field tuning was finally performed to a level of $\sigma\left(B_{y, 0}\right) \sim 1 \%$. First and second field integrals, representing the deflection strength and beam path, were cross-checked with the pulsed-wire method [18] during the tuning procedure. These measurements [19] exhibited a minor deflection of 400-800 MeV electron beams behind the undulator of few $10 \mu \mathrm{rad}$. In the experiments at the MAMI facility, described below, no beam deflection was visible on a screen some $10 \mathrm{~m}$ downstream, confirming the order of magnitude. Phase shake, defined as the deviation from the mean phase for one fixed point every half period [20], amounts to $\Delta \Psi_{\text {rms }}=2.2^{\circ}$ and corresponds to a relative phase error of less than $1 \%$.

In order to later investigate the effects of surface current induced wakefields on intense laser-accelerated electron bunches [8], the gap height of the prototype undulator can be varied over a wide range. Starting at the lowest value that allows for reasonable electron beam transmission of $g=1.6 \mathrm{~mm}\left(g / \lambda_{u}=0.32\right)$, a peak field of $B_{y, 0}=0.91 \mathrm{~T}$ was achieved. It exceeds that of typical $\mathrm{cm}$-scale hybrid designs (of same $g / \lambda_{u}$ ) by a few percent [9] and corresponds to an undulator parameter of $K=0.42$. With increasing $g$, the near exponential decrease of the peak field [9], depicted in Fig. 5, nicely corresponds to the design simulation and allows for a wide range of operation.

\section{B. Performance tests}

Present laser-driven accelerators still exhibit considerable pulse-to-pulse fluctuations in energy and directional pointing stability. Two complementary performance
TABLE I. Undulator, electron beam (energy, unnormalized typical emittances, measured beam radii, and divergences at the position of the undulator), and radiation parameters for the two independent performance tests of the undulator at the Mainz microtron as described in the text.

\begin{tabular}{lccc}
\hline \multicolumn{1}{c}{$K$} & 0.32 & 0.45 & 0.45 \\
\hline$E_{e}[\mathrm{MeV}]$ & 855 & 705 & 405 \\
$\varepsilon_{x}\left[10^{-9} \mathrm{~m} \mathrm{rad}\right]$ & 10 & 5 & 2 \\
$\varepsilon_{y}\left[10^{-9} \mathrm{~m} \mathrm{rad}\right]$ & 0.5 & 0.5 & 0.8 \\
$\sigma_{x}[\mu \mathrm{m}]$ & 510 & 370 & 236 \\
$\sigma_{y}[\mu \mathrm{m}]$ & 200 & 55 & 37 \\
$\sigma_{x}^{\prime}[\mu \mathrm{rad}]$ & & 53 & \\
$\sigma_{y}^{\prime}[\mu \mathrm{rad}]$ & & 3 & \\
\hline$n$ & $1,2,3,4$ & 1 & 3 \\
$E_{n}[\mathrm{keV}]$ & $($ simultaneously $)$ & & \\
\hline \hline
\end{tabular}

tests of the miniature undulator have therefore been pursued at the Mainz microtron facility MAMI [21]. Table I summarizes the corresponding beam parameters.

The aim of the first test experiment at MAMI was the simultaneous characterization of the spatial emission pattern of the undulator radiation for different harmonic numbers $n$. At an electron energy of $855 \mathrm{MeV}(\gamma=1674)$, an undulator parameter of $K \sim 0.32(g \sim 2 \mathrm{~mm})$, and zero emission angle, a photon energy of the fundamental of $E_{n=1} \sim 1.32 \mathrm{keV}$ was expected. Radiation energy spectra could thus be directly observed with a cold Ge detector providing a $200 \mathrm{eV}$ (FWHM) energy resolution in single photon counting mode. The detector was covered with an aperture of $250 \mu \mathrm{m}$ radius and positioned $8.8 \mathrm{~m}$ downstream, so that an angular resolution of $30 \mu \mathrm{rad}$ was achieved. This resolution was chosen to be of the order of the expected rms spread of the fundamental radiation $(n=1)$ of a perfect planar undulator,

$$
\theta_{\mathrm{rms}, x} \sim \frac{1}{2 \gamma} \sqrt{\frac{1+K^{2} / 2}{n N_{u}}} \sim \frac{1}{2 \gamma \sqrt{N_{u}}},
$$

giving $\theta_{\mathrm{rms}, x} \sim 40 \mu \mathrm{rad}$ for $N_{u}=60$. Electron beam transport was adjusted in order to provide a nearly collimated beam at the position of the undulator. Wire scan measurements at the entrance and exit of the undulator revealed rms widths of $\sigma_{x}=0.5 \mathrm{~mm}$ and $\sigma_{y}=0.2 \mathrm{~mm}$. Thus, regarding a horizontal emittance of the order of $10^{-8} \mathrm{~m} \mathrm{rad}$, the contribution of the remaining electron beam divergence to the emission angle of the undulator radiation remains small for this experiment.

Figure 6 shows the measured energy spectra of the undulator radiation as a function of the horizontal and vertical emission angles, where the general angular shape is described by Eq. (1) (solid red lines in the density plots). On axis ( $\theta=0$, upper left figure), the total yield is compared to simulations (red line) using the single particle 

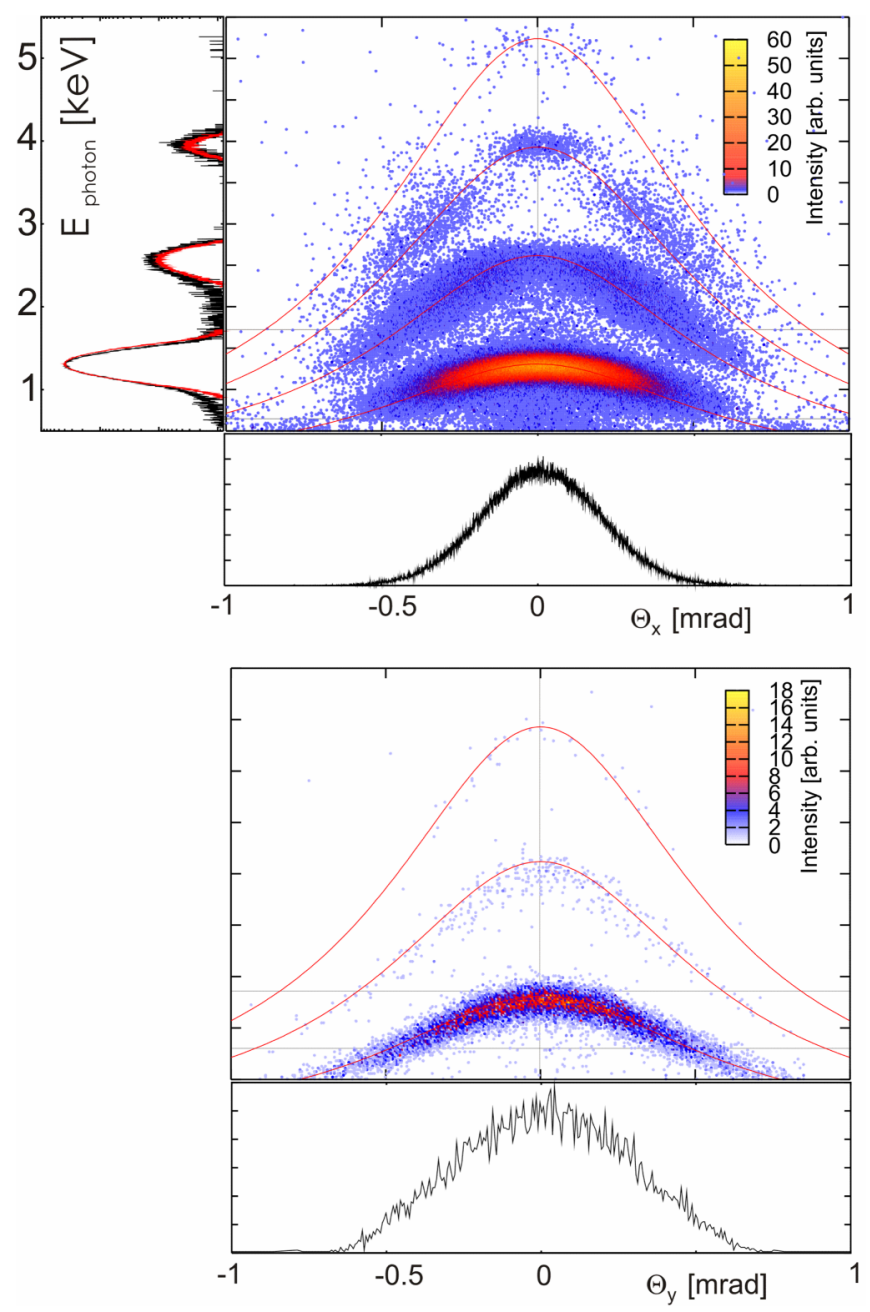

FIG. 6. (Color) Undulator radiation spectra as a function of the horizontal $(x)$ and the vertical $(y)$ observation angles $\theta$, recorded with a Ge detector at an electron energy of $855 \mathrm{MeV}$ at MAMI. The absolute on-axis yield for harmonics up to the third is compared to SPECTRA simulations [22] in the left graph (red line) including an energy resolution of $200 \mathrm{eV}$ (FWHM). The angular spread, integrated between the thin horizontal lines, is depicted in the lower one. Solid (red) lines indicate the general $\gamma^{2} \theta^{2}$ dependence of the energy.

code SPECTRA [22], including aperture and energy resolution of the detector. Good agreement is achieved, especially in the amplitude ratio between different harmonics, while details of the spectrum are blurred due to detector resolution.

The second experiment aimed for the precise measurement of the energy spectrum. More stringently than the previous measurement, it provides information on the number of undulator periods for which the undulator radiation adds up coherently and thus on the quality of the beam path inside the undulator.

For the case of an ideal undulator, the radiation is emitted within the box-shaped time window corresponding to the passage of an electron through the alternating field.
Thus, the on-axis energy spectrum is described by its Fourier-transform

$$
I_{n}(E) \propto \sin ^{2}\left(\pi n N_{u} \frac{E_{n}-E}{E_{n}}\right) /\left(\pi n N_{u} \frac{E_{n}-E}{E_{n}}\right)^{2}
$$

and the relative bandwidth (FWHM) of the different harmonics can be approximated by

$$
\frac{\Delta E_{n}}{E_{n}} \sim \frac{2 \times 1.4}{\pi n N_{u}} \sim \frac{1}{n N_{u}} .
$$

For this experiment, a high-resolution grating spectrometer with variable line spacing was installed instead of the Ge detector. It was read out by an efficient x-ray CCD camera (pixel size $13 \times 13 \mu \mathrm{m}^{2}$ ) some $6.7 \mathrm{~m}$ further downstream $[11,23]$. Succeeding the first experiment, the undulator was carefully retuned to the most critical minimum gap height of $g \sim 1.5 \mathrm{~mm}(K=0.45)$. Pulsed-wire measurements indicated a straight electron trajectory in the sense that deviations from the mean path are small compared to the deflection amplitude of the undulating beam over at least the inner $90 \%$ of the path [19]. The electron beam energy was set to 705 and $405 \mathrm{MeV}$ as these energies lead to a radiation energy of $E_{n} \sim 860 \mathrm{eV}$ for the fundamental and the third harmonic, respectively. This photon energy coincides with two $L$-absorption edges of a thin $\mathrm{Ni}$ foil $\left(E_{L 2}=854.7 \mathrm{eV}, E_{L 3}=871.9 \mathrm{eV}\right.$ [23]) providing the required high-resolution online calibration of the linewidth of the undulator radiation. Electron beam focusing was optimized for a minimum linewidth taking into account beam divergence as well as the analytical off-axis field distribution of an undulator.

Figure 7 depicts energy spectra of the undulator radiation on axis $(\theta=0)$ for the fundamental and for the third harmonic, that are extracted from images resolving the horizontal angular distribution [19]. The measured relative

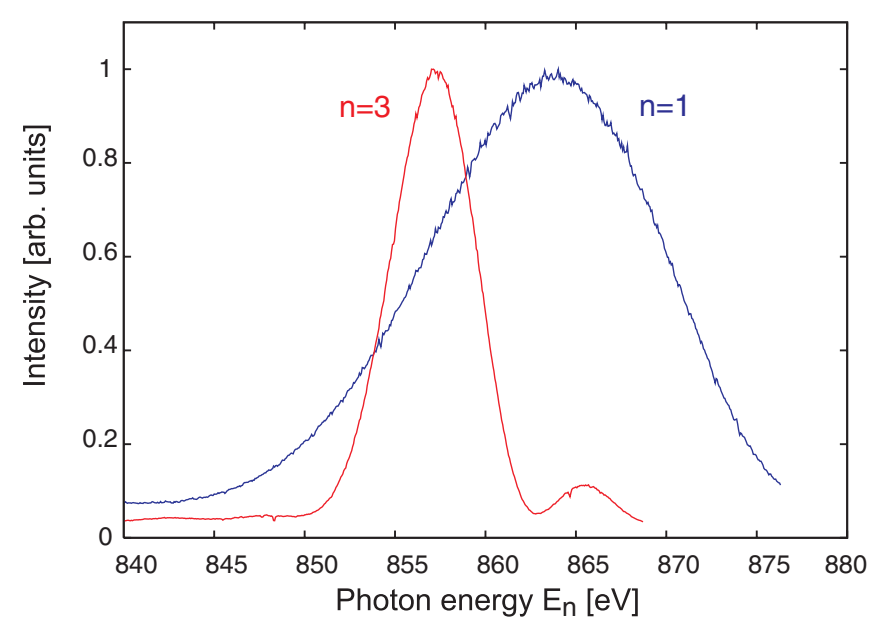

FIG. 7. (Color) On-axis high-resolution energy spectra of the fundamental $(n=1)$ and the more narrow third harmonic $(n=$ 3 ) undulator radiation for an electron energy of 705 and $405 \mathrm{MeV}$, respectively. 
width (FWHM) of the spectra of $\Delta E_{1} / E_{1}=0.0174$ and $\Delta E_{3} / E_{3}=0.0063$, respectively, translates into an effective number of undulator periods of $N_{u \text {,eff }} \sim 53-58$, when the approximate Eq. (5) is applied. A further consideration of the measured field and phase fluctuations consistently reveals the participation of the full undulator $\left(N_{u \text {,eff }} \sim 60\right)$ in the generation of the radiation and thus confirms its design performance.

\section{COMPACT HIGH-GRADIENT QUADRUPOLE MAGNETS}

Beam transport and especially imaging from the compact laser-plasma accelerator to the miniature undulator has to be provided by compact devices with short focal length. The construction of such compact quadrupole magnets with high field gradients relies on well-established principles. On the one hand, the absolute field on the magnet poles $B\left(r_{i}\right)$ has to be maximized while, on the other, the bore radius $r_{i}$ has to be minimized. A reasonable compromise can be achieved for $r_{i}=3 \mathrm{~mm}$ and $B\left(r_{i}\right) \sim$ $1.5 \mathrm{~T}$ leading to a gradient of the order of $500 \mathrm{~T} / \mathrm{m} \mathrm{[12].}$ Here, a simple and economical design has been chosen, where the quadrupole field is defined by four radially magnetized wedges of $\mathrm{Nd}_{2} \mathrm{Fe}_{14} \mathrm{~B}$ magnets [15]. The outer field closure is accomplished by four additional pairs of permanent magnets as sketched in Fig. 8. As the four main wedges are strongly attracted toward the center of the quadrupole, mechanical precision and thus field accuracy can be achieved by the insertion of a nonmagnetic precision cylinder into the center of the quadrupole, as visible in the photograph in Fig. 9. Three-dimensional field simulations (CST EM Studio) as well as electron tracking simulations revealed a sufficient optical quality of this simple

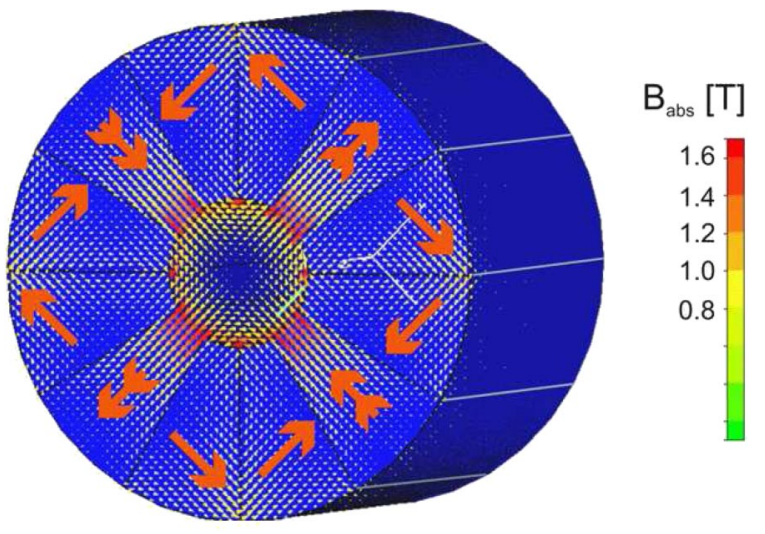

FIG. 8. (Color) Schematic view of the 12 segment Halbach design of one quadrupole magnet. While 4 radially magnetized segments define the quadrupole field, 8 are used for the outer field closure, thick arrows indicating the direction of magnetization. False color arrows illustrate the simulated strength and direction of the resulting quadrupole field inside a bore of radius $r_{i}=3 \mathrm{~mm}$. The tip field strength amounts to $B\left(r_{i}\right) \sim 1.5 \mathrm{~T}$.

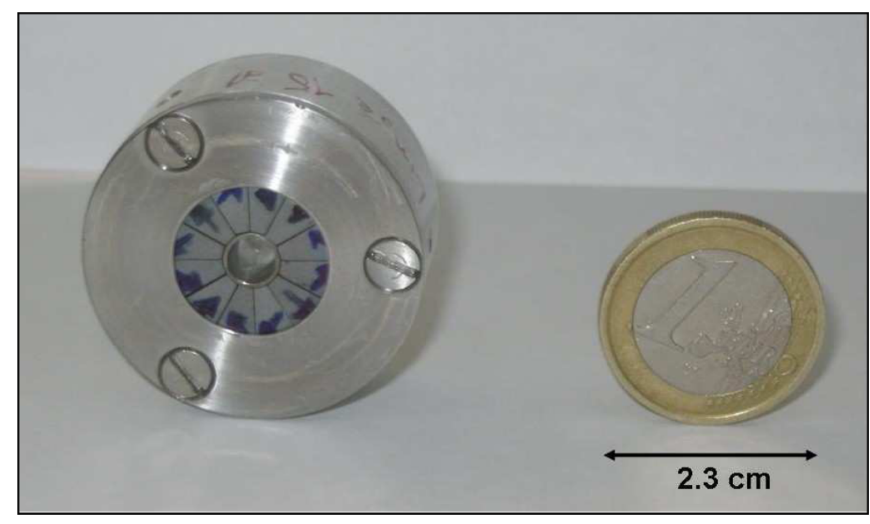

FIG. 9. (Color) Photograph of one mounted miniature quadrupole magnet.

design, comparable to the more complex one recently realized by the PLEIADES group [12].

The field gradient has been verified by systematic measurements of the absolute field as a function of the distance from the quadrupole center. As for the case of the undulator, a precision Hall probe with a head of $0.75 \mathrm{~mm}$ diameter was used. Ideally, the probe size should not affect the measurement. Figure 10 expresses not only the good agreement between the design simulations and the measurement, but also the integrity of the quadrupole field over almost the whole aperture of the quadrupole. Ten different quadrupole magnets were analyzed, leading to an average field gradient of $(503 \pm 6) \mathrm{T} / \mathrm{m}$.

In order to further investigate potential effects of deviations from an ideal quadrupole field, single quadrupoles as well as doublets were tested at the Mainz microtron facility MAMI. An electron energy of $600 \mathrm{MeV}$ was chosen, where the unnormalized rms emittance of the beam amounts to $\varepsilon_{y}=0.5 \times 10^{-9} \mathrm{~m} \mathrm{rad}$ and the relative energy spread to

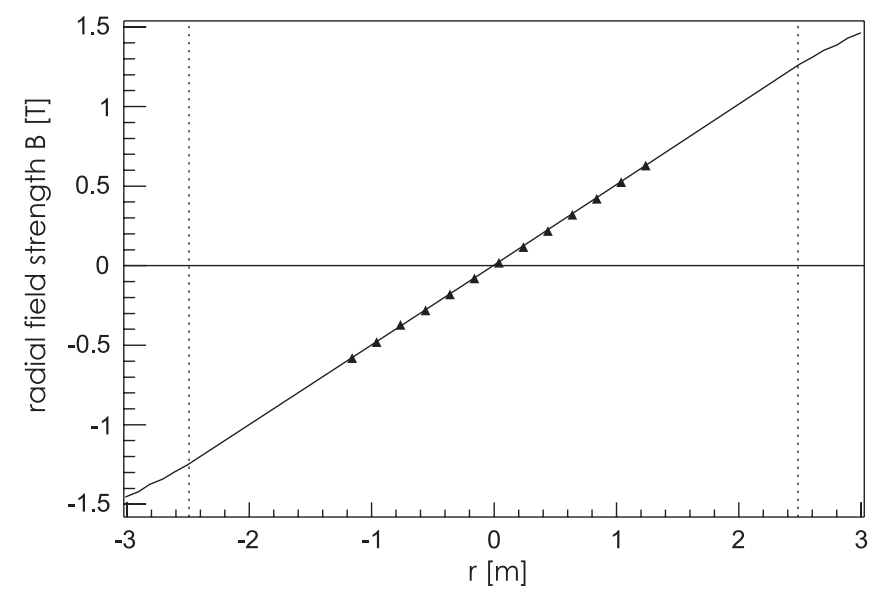

FIG. 10. Hall probe measurements (triangles) are plotted against the distance from the center of the quadrupole and compared to the design simulation (solid line). The dashed lines indicate aperture limitations originating from the central nonmagnetic cylinder. The field gradient amounts to $503 \mathrm{~T} / \mathrm{m}$. 
$10^{-5}$. The incoming beam was nearly collimated for an illumination of a large fraction of the lens. Beam radii were again measured by scanning tungsten wires of 4 and $10 \mu \mathrm{m}$ diameter through the low current electron beam. In Fig. 11 the beta function of the electron beam $\beta_{y}(z)=$ $\sigma_{y}^{2}(z) / \varepsilon_{y}$, derived from the measured envelope radius $\sigma_{y}$, is depicted around the focus $\sim 15 \mathrm{~cm}$ behind the quadrupole magnet. For simplicity only one quadrupole was used, and therefore the vertical degree of freedom was focused to a line focus. From the parabolic shape around the focal point (introducing the relative position coordinate $s$ ), the beta function at the focal point $\beta_{y}^{*}$

$$
\beta_{y}(s)=\beta_{y}^{*}+\frac{s^{2}}{\beta_{y}^{*}}
$$

can be estimated to $\beta_{y}^{*} \sim 1.2 \mathrm{~mm}$, yielding a width of the focal line of the order of below one micron.

The value of the beta function turns out to be in good overall agreement with the Hall probe measurements, the
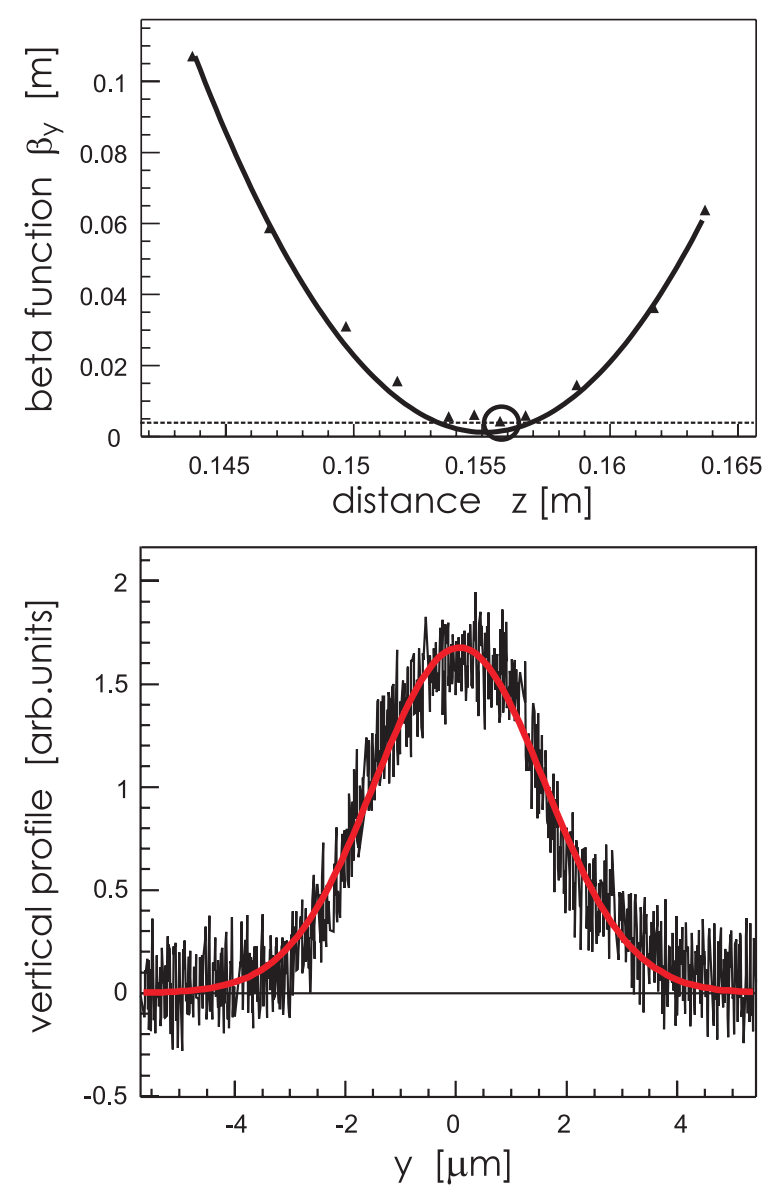

FIG. 11. (Color) Results of the measurement of the imaging properties of a single quadrupole magnet. The triangles in the upper graph depict the measured beta function. The horizontal line indicates the resolution of a single measurement due to the thickness of the wire of $4 \mu \mathrm{m}$. The lower graph illustrates the resolution limited width measured close to the focal point. measured beam size in front of the quadrupole magnet and the remaining beam divergence. We therefore conclude that the imaging properties of this simple lens are sufficient for both the laser-plasma applications as well as for the future generation of a two-dimensional sub-micron focal spot at MAMI.

\section{STATUS AND PERSPECTIVES}

Having demonstrated the performance of the miniaturized magnetic devices, the following section focuses on applications with laser-plasma accelerated electron beams, constraints that might arise due to the high peak currents, and technological perspectives for even better performance at similar dimensions.

One first application of miniature undulators will be their use as a diagnostic tool for laser-accelerated electron beams. For laser-plasma accelerator laboratories compact electron spectrometers, that do not rely on magnetic dispersion, are highly valuable. Usually, space is too restricted for the installation of dipole magnets with bending radii of the order of $10 \mathrm{~m}$ for the detection of electrons of $\mathrm{GeV}$ energies. A well characterized diagnostic undulator, however, could be used for measuring electron energy and energy spread as well as the beam divergence. Laseraccelerated electron beams typically exhibit broad spectra of the order of $1 \%-10 \%$. Thus, an intrinsic line width $1 /\left(n N_{u}\right)$ of $<1 \%$ is sufficient to determine the energy spread with unprecedented accuracy. Beam alignment errors and divergence have to be kept small so that according to Eq. (1) only the variation in $\gamma$ effects the observed wavelength $\lambda_{1}$. On the other hand, the electron beam divergence can be measured provided the divergence of the undulator light $1 /\left(2 \gamma \sqrt{n N_{u}}\right)$ is significantly smaller. Both conditions imply that a diagnostic undulator must have a period number of at least $N_{u} \sim 100$.

Regarding the potential for high peak currents of $I \sim$ $100 \mathrm{kA}$ [6] in laser-plasma acceleration for energies of presently up to $\sim \mathrm{GeV}$, miniature undulators should be used in laser-driven FELs in order to reach the regime of extreme UV and soft x-ray radiation. Such a regime [8] leads to comparatively large Pierce parameters $\rho$ and hence higher tolerance of the FEL process against disturbing effects, such as initial energy spread and emittance, but also wakefield-induced energy spreads [24]. Further, for medical applications, where photons of at least $20 \mathrm{keV}$ are required, quantum fluctuations in the spontaneous emission [25] cause additional energy spread which hinders the FEL process. Since their impact increases with $\gamma$ as $d\left\langle\Delta \gamma^{2}\right\rangle / d z \propto \gamma^{4}$, electron energies should remain below $10 \mathrm{GeV}$. Note that, for the generation of $50 \mathrm{keV}$ photons, $7 \mathrm{GeV}$ electrons and an undulator period of $7 \mathrm{~mm}$ are sufficient.

A short undulator period must thus be compensated by peak currents $I$ that exceed those used in large-scale FEL facilities. On the one hand, a large Pierce parameter re- 
duces the saturation length of the FEL. On the other, the saturation power of the FEL $P_{\text {sat }} \propto \rho P_{\text {beam }}$ roughly scales as $P_{\text {sat }} \propto\left(I \lambda_{u}\right)^{4 / 3}$ [8]. However, the shorter the undulator period is, the smaller the corresponding gap height becomes and thus, the stronger the induced wakefields. This effect constitutes a fundamental limit to the undulator period besides the technological obstacles.

The undulator presented in this paper was found to have a mean field inhomogeneity of about $1 \%$. Since the undulator parameter $K=0.4$ remains below unity, the effective increase of the undulator radiation wavelength $\Delta \lambda_{1} / \lambda_{1}$ is smaller by a factor of $K^{2} \Delta K / K$, hence $\Delta \lambda_{1} / \lambda_{1}=1.6 \times$ $10^{-3}$. Since the Pierce parameter amounts to about 0.01 for the proof-of-principle FEL suggested in Ref. [8], the present field errors appear tolerable. Although the present undulator is about a factor of 3 too short for reaching saturation of the SASE process, amplification should become visible $[8,26]$. The undulator radiation spectra measured at MAMI exhibit a well-reproducible ratio of the intensity of the fundamental and the third harmonic in the case of spontaneous emission. For the detection of SASE signatures this ratio is important, because a change in favor of the fundamental constitutes a hint for amplification.

Regarding the high peak currents, resistive wall and geometric wakefields seem likely to represent the main degradation effect for table-top FELs. The most obvious countermeasure will be an increase of the undulator gap size as the strength of the wakefields scales as one over the square of the gap size. However, merely increasing the gap would lower the peak magnetic field strength on axis and thus the $K$ parameter. The intensity of spontaneous undulator radiation scales with $K^{2}$ and, furthermore, a decrease in $K$ leads to an increase in the FEL saturation length. Thus, an increase of the gap size can only be accepted when novel techniques are developed that maintain the field strength on axis.

Future progress in the increase of the peak field of miniature undulators by about $30 \%$ could be made by cooling the permanent magnets to near liquid nitrogen temperatures. It has been suggested that for hybrid types the saturation field strength in the ferromagnetic pole material can be increased by simultaneously replacing the Co steel by dedicated materials such as dysprosium [27]. Superconducting technology on the other hand has not yet reached $\mathrm{mm}$-scale period length with the desired field strength. The smallest prototype of the Karlsruhe group $\left(\lambda_{u}=3.8 \mathrm{~mm}\right)$ based on NbTi wires reached peak fields of around $1 \mathrm{~T}$ for a gap of $1 \mathrm{~mm}$ [28]. Recent results achieved [29] with an in-vacuum, $\lambda_{u}=14 \mathrm{~mm}$, and $g / \lambda_{u}=0.6$ undulator indicate an improvement of about $20 \%$ compared to conservative hybrid designs and no improvement over the one presented here. However, there is room for future coil development based on $\mathrm{Nb}_{3} \mathrm{Sn}$ that might allow for comparative $K$-values and the desired increase in the gap height [30] by a factor of about 1.5 .
Briefly summarizing, we presented results on the successful commissioning of miniaturized lenses and undulators dedicated for the use in the field of laser-plasma accelerators. First experiments on beam diagnostics in this field are presently on the way and further undulator development as mentioned above is planned.

\section{ACKNOWLEDGMENTS}

This work has been funded by DFG through TRANSREGIO TR18 and supported by the DFG clusterof-excellence Munich Center for Advanced Photonics MAP.

[1] S. P. D. Mangles, C. Murphy, Z. Najmudin, A. Thomas, J. Collier, A. Dangor, E. Divall, P. Foster, J. Gallacher, C. Hooker, D. Jaroszynski, A. Langley, W. Mori, P. Norreys, F. Tsung, R. Viskup, B. Walton, and K. Krushelnick, Nature (London) 431, 535 (2004).

[2] C. G. R. Geddes, C. Toth, J. van Tilborg, E. Esarey, C. Schroeder, D. Bruhwiller, C. Nieter, J. Cary, and W. Leemans, Nature (London) 431, 538 (2004).

[3] J. Faure, Y. Glinec, A. Pukhov, S. Kiselev, S. Gordienko, E. Lefebvre, J.-P. Rousseau, F. Burgy, and V. Malka, Nature (London) 431, 541 (2004).

[4] W. P. Leemans, B. Nagler, A. Gonsalves, C. Toth, K. Nakamura, C. Geddes, E. Esarey, C. Schroeder, and S. Hooker, Nature Phys. 2, 696 (2006).

[5] J. Faure, C. Rechatin, A. Norlin, A. Lifschitz, Y. Glinec, and V. Malka, Nature (London) 444, 737 (2006).

[6] M. Geissler, J. Schreiber, and J. Meyer-ter-Vehn, New J. Phys. 8, 186 (2006).

[7] A. Rousse, K. T. Phuoc, R. Shah, A. Pukhov, E. Lefebvre, V. Malka, S. Kiselev, F. Burgy, J.-P. Rousseau, D. Umstadter, and D. Hulin, Phys. Rev. Lett. 93, 135005 (2004).

[8] F. Grüner, S. Becker, U. Schramm, T. Eichner, M. Fuchs, R. Weingartner, D. Habs, J. Meyer-ter-Vehn, M. Geissler, M. Ferrario, L. Serafini, B. an der Geer, H. Backe, W. Lauth, and S. Reiche, Appl. Phys. B 86, 431 (2007).

[9] P. Elleaume, J. Chavanne, and B. Faatz, Nucl. Instrum. Methods Phys. Res., Sect. A 455, 503 (2000).

[10] R. Bonifacio, C. Pellegrini, and L. M. Narduci, Opt. Commun. 50, 373 (1984).

[11] M. El-Ghazaky, H. Backe, W. Lauth, G. Kube, P. Kunz, A. Sharafutdinov, and T. Weber, Eur. Phys. J. A 28, 197 (2006).

[12] J. K. Lim, P. Frigola, G. Travish, J. B. Rosenzweig, S. G. Anderson, W. J. Brown, J. S. Jacob, C. L. Robbins, and A. M. Tremaine, Phys. Rev. ST Accel. Beams 8, 072401 (2005).

[13] J. Meyer-ter-Vehn and A. Pukhov, Appl. Phys. B 74, 355 (2002).

[14] K. Halbach, Nucl. Instrum. Methods 187, 109 (1981).

[15] Vacuumschmelze Hanau, Vacoflux 50, and Vacodym 764 HR data sheets, 2005, http://www.vacuumschmelze.com.

[16] S. Dambach, H. Backe, Th. Doerk, N. Eftekhari, H. Euteneuer, F. Görgen, F. Hagenbuck, K. H. Kaiser, 
O. Kettig, G. Kube, W. Lauth, H. Schöpe, A. Steinhof, Th. Tonn, and Th. Walcher, Phys. Rev. Lett. 80, 5473 (1998); S. Dambach, Ph.D. thesis, Universität Mainz, 1998.

[17] I. Vassermann and E. R. Moog, Rev. Sci. Instrum. 66, 1943 (1995).

[18] R. W. Warren, Nucl. Instrum. Methods Phys. Res., Sect. A 272, 257 (1988).

[19] T. Eichner, Diploma thesis, LMU Munich, 2007.

[20] R. P. Walker, Nucl. Instrum. Methods Phys. Res., Sect. A 335, 328 (1993).

[21] http://www.kph.uni-mainz.de/B1/floorplan.php.

[22] T. Tanaka and H. Kitamura, J. Synchrotron Radiat. 8, 1221 (2001).

[23] W. Lauth, H. Backe, O. Kettig, P. Kunz, A. Sharafutdinov, and T. Weber, Eur. Phys. J. A 28, 185 (2006).

[24] A.W. Chao, Physics of Collective Beam Instabilities in High Energy Accelerators (John Wiley \& Sons, New York,
1993); K. Bane, SLAC-PUB-11829, 2006.

[25] M. Sands, Phys. Rev. 97, 470 (1955).

[26] S. Reiche, Nucl. Instrum. Methods Phys. Res., Sect. A 429, 243 (1999).

[27] T. Hara, T. Tanaka, H. Kitamura, T. Bizen, X. Marechal, T. Seike, T. Kohda, and Y. Matsuura, Phys. Rev. ST Accel. Beams 7, 050702 (2004).

[28] T. Hezel, B. Krevet, H. O. Moser, J. A. Rossmanith, R. Rossmanith, and T. Schneider, J. Synchrotron Radiat. 5, 448 (1998).

[29] S. Casalbuoni, M. Hagelstein, B. Kostka, R. Rossmanith, M. Weisser, E. Steffens, A. Bernhard, D. Wollmann, and T. Baumbach, Phys. Rev. ST Accel. Beams 9, 010702 (2006).

[30] R. Rossmanith (private communication). 